\title{
Síndrome de pseudo-Duane secundario a Schwannoma de nervio abducens: Reporte de caso y revisión
}

\author{
Pseudo-Duane syndrome secondary to abducens nerve schwannoma: Case report and review
}

\author{
Juan Carlos Bustos Orellana', Cristian Salgado Alarcón', Sebastián Bravo-Grau²
}

\section{Resumen}

Reporte de caso de síndrome de pseudo-Duane asociado a schwannoma de nervio abducens y neurofibromatosis tipo 2 (NF2). Este síndrome es raro, caracterizado por limitación en abducción, retracción ocular, disminución de hendidura palpebral en abducción y restricción del recto medial ipsilateral en test de ducción forzada. La NF2 es también una enfermedad infrecuente caracterizada por tumores de sistema nervioso central y periférico. Los schwannomas de nervio abducens son también poco frecuentes, y se presentan comúnmente con diplopia. Se revisan brevemente estas enfermedades. Es el primer caso reportado de pseudo-Duane secundario a schwannoma de nervio abducens y NF2 según nuestro conocimiento.

Palabras clave: pseudo-Duane; neurofibromatosis 2; schwannoma; nervio abducens; estrabismo; diplopía.

\begin{abstract}
A pseudo-Duane syndrome case associated with abducens nerve schwannoma and neurofibromatosis type 2 (NF2) is presented. This syndrome is a rare disease characterised by abduction limitation, ocular retraction, narrowing of the palpebral fissure in abduction, and ipsilateral medial rectus restriction on forced duction test. NF2 is also an uncommon disease which is characterised by peripheral and central nervous system tumours. Abducens nerve schwannomas are also uncommon and presents usually as diplopia. A short review of these diseases is given. This is the first case of pseudo-Duane secondary to abducens nerve schwannoma, to our knowledge.
\end{abstract}

Keywords: pseudo-Duane's syndrome; neurofibromatosis 2; schwannoma; abducens nerve; strabismus; diplopia.

Fecha de envío: 2020-11-11 - Fecha de aceptación: 2021-03-16

\section{Introducción}

El síndrome de pseudo-Duane fue descrito en 1976 por Duane y Schatz. Es también conocido como síndrome de Duane inverso, reverso, restrictivo o en espejo. Es una enfermedad rara que se caracteriza por limitación en abducción, retracción ocular, disminución de hendidura palpebral en abducción, y restricción de recto medio ipsilateral en test de ducción forzada. La etiología de este síndrome se ha descrito como secundaria a trauma (Duane et al., 1976; Gittinger et al., 1986; Pelit, 2016; Hee et al., 2009), acortamiento congénito de recto medial (Lew et al., 2000), banda fibrosa entre recto medial y pared orbitaria (Chatterjee et al., 1991), cicatrización postquirúrgica (pterigión (Duane et al., 1976; Khan, 2005), dermolipoma (Von Noorden, 1980), miocisticercosis
(Murthy, 2008), metástasis de cáncer de mama (Duane et al., 1976; síndrome paraneoplásico (Cabrillo et al., 2010). Se presenta un caso de pseudo-Duane de origen tumoral.

\section{Caso clínico}

Se presenta a la consulta un paciente de sexo masculino de 20 años de edad, con historia de hamartomas de cuero cabelludo removidos quirúrgicamente en 2 ocasiones, sin otros antecedentes mórbidos. Refiere 5 años de diplopia horizontal en la mirada hacia la derecha y posición anormal de cabeza. Al examen físico, presenta agudeza visual mejor corregida logMAR 0,04 (20/22 Snellen) en ojo derecho y logMAR 0,1 (20/25 Snellen) en ojo izquierdo, motilidad con limitación del $80 \%$ en abducción del ojo derecho (Figura 1),

(1) Departamento de Oftalmología, Pontificia Universidad Católica de Chile. 
disminución de la hendidura palpebral en aducción, cover test con endotropia (ET) derecha que alterna con ET izquierda, test de Hirschberg evidencia una leve ET de $3^{\circ}$, giro de cabeza a izquierda con ortotropia en su posición anómala. Visión de colores y reflejos fotomotores normales en ambos ojos. En polo anterior destaca catarata polar posterior central pequeña en ojo derecho. Fondo de ojo con nervio óptico de aspecto normal, sin otros hallazgos patológicos. Se solicita resonancia nuclear magnética (RNM) para descartar patología secundaria, que evidencia schwannomas en nervio abducens derecho (Figura 2), nervio vestibular y meningioma espinal en $\mathrm{C} 1$, que configuran un cuadro radiológicamente compatible con neurofibromatosis tipo 2 (NF2).
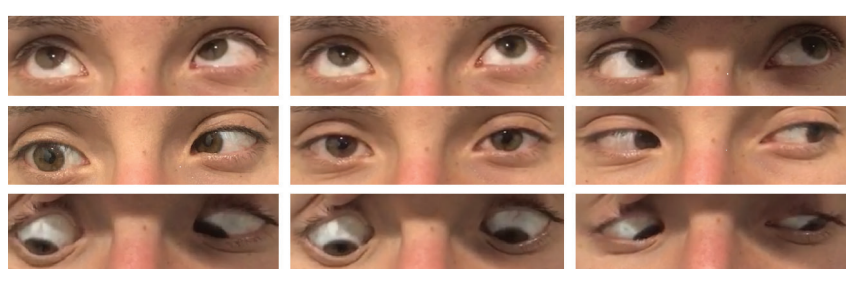

Figura 1: Fotografía de posiciones de mirada. Se nota mínima endotropia en posición primaria de mirada, limitación del $80 \%$ en abducción del ojo derecho y disminución de hendidura palpebral en aducción en ojo derecho.

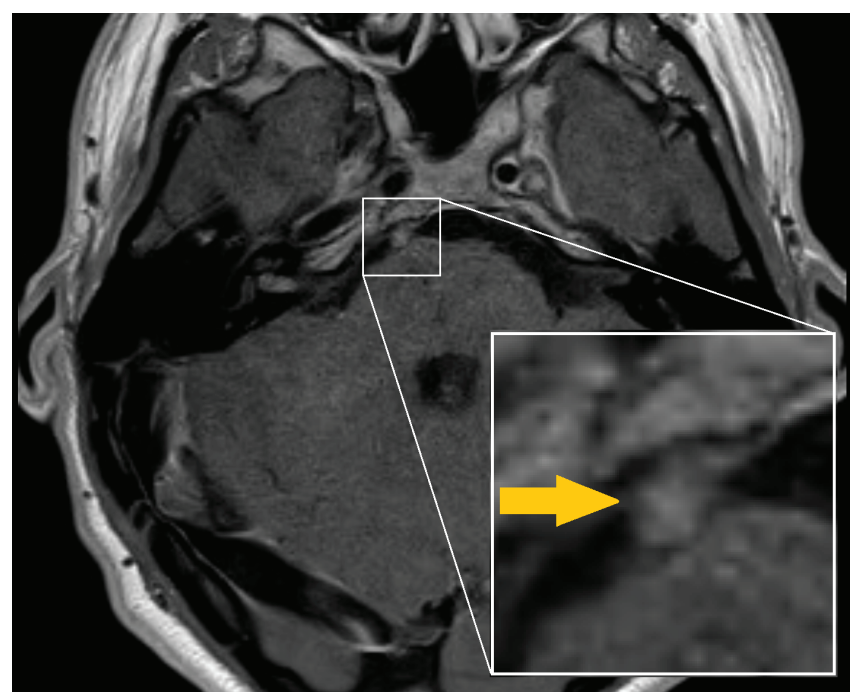

Figura 2: Secuencia T1 de resonancia nuclear magnética de cerebro. Se identifica un tumor ovoídeo en nervio abducens derecho en su posición cisternal.

\section{Discusión}

NF2 es una enfermedad rara, autosómica dominante. Mutaciones esporádicas también han sido identificadas. Hay una mutación del gen NF2 es un gen supresor de tumores, localizado en el brazo largo del cromosoma 22, que codifica para la proteína merlina. Esto lleva a una señal mitogénica descontrolada y a génesis de tumores. Se presenta típicamente con sordera y ciertos hitos radiológicos como schwannomas vestibulares bilaterales junto a otros tumores de sistema nervioso central y periférico (Von Noorden, 1980). Entre sus manifestaciones oculares encontramos cataratas (subcapsulares posteriores y corticales periféricas) y membrana epirretinal (Kaiser-Kupfer et al., 1989; Bosch et al., 2006). Hallazgos menos comunes son meningiomas de nervio óptico (Bosch et al., 2006), gliomas de disco óptico (Dossetor et al., 1989), hamartomas combinados de epitelio pigmentario retinal y retina (Sivalingam et al. 1990), nódulos de Lisch y anormalidades de nervios craneales. Todos los nervios craneales pueden estar comprometidos, y esto podría manifestarse como estrabismo (Tonsgard \& Oesterle, 1993; Feucht et al., 2008), pérdida de visión o queratopatía neurotrófica. El estrabismo puede ser de cualquier tipo, tanto comitante como no comitante (Feucht et al., 2008). RNM es el método de imagen de elección para el estudio de pacientes con sospecha de NF2.

Los schwannomas, por otra parte, son tumores que comprometen las células de Schwann de las neuronas mielinizadas. Estos pueden identificarse como masas focales de crecimiento excéntrico bien definidas desde un nervio craneal (Rato et al., 2012). Schwannomas de nervios oculomotores son raros, y de ellos los que afectan el nervio abducens son los menos comunes, comprendiendo el $13 \%$ de ellos (Nakamura et al., 2002). Estos se presentan en 3 escenarios: aislados, asociados a neurofibromatosis o asociados a schwannomatosis (Rato et al., 2012). Usualmente estos casos se presentan como una paresia de sexto nervio craneal.

Los schwannomas de nervio abducens han sido clasificados por Tung (Tung et al., 1991) basados en su localización anatómica y sus síntomas y se dividen en tipo 1 a aquellos ubicados en el seno cavernoso, generalmente asociados a paresia de abducens y cefalea; y tipo 2 que se localizan en el área prepontina con síntomas de hipertensión intracraneal. Casos muy raros de schwannomas de nervio abducens intraorbitarios se han reportado. El uso de neuroimágenes es muy útil para su diagnóstico, pero su diagnóstico final está basado en la histología y en la respuesta clínica a la cirugía ( $\mathrm{Li}$ et al., 2015). Casos extracavernosos se han resecado por distintas vías, generalmente por un abordaje retrosigmoídeo suboccipital lateral simple, con recuperación potencial de la función nerviosa (Lida et al., 2016).

\section{Conclusión}

Finalmente, nuestro paciente decide no intervenir de momento su estrabismo ni sus tumores, ya que no presenta otros síntomas y ni la diplopia ni el giro de cabeza afectan significativamente su calidad de vida. Aunque no hemos podido confirmar este caso histológicamente, quirúrgicamente o por respuesta clínica postoperatoria, las neuroimágenes y el cuadro clínico son altamente compatibles con el diagnóstico. Este es un cuadro atípico de síndrome de 
pseudo-Duane especialmente por la disminución de hendidura palpebral en aducción en vez de abducción. Es importante mencionar que la prueba de ducción forzada no se realizó en nuestro paciente pues no se intervino quirúrgicamente, ya que el mejor contexto para su realización es bajo anestesia general. Este caso corresponde, a nuestro conocimiento, el primer caso reportado de esta condición secundaria a schwannoma de nervio craneal asociado a NF2.

\section{Contribuciones y reconocimientos}

Juan Carlos Bustos realizó la evaluación clínica, redacción y revisión bibliográfica, Cristian Salgado la evaluación clínica, supervisión y corrección y Sebastián Bravo-Grau la evaluación clínica imagenológica.

Los autores no presentan algún conflicto ético y el trabajo se realizó sin fuentes de financiamiento.

\section{Referencias}

Bosch MM, Boltshauser E, Harpes P \& Landau K. (2006). Ophthalmologic findings and long-term course in patients with neurofibromatosis type 2. American Journal of Ophthalmology 141, 1068-77.

Cabrillo L, Lorenzo R, de Juan L, Cruz F \& Hernández E. (2014). Síndrome de pseudo-Duane paraneoplásico secundario a tumor de mama. Revista Española de Investigaciones Oftalmológicas 4, 35-7.

Chatterjee PK, Bhunia J \& Bhattacharyya I. (1991). Bilateral inverse Duane's retraction syndrome-a case report. Indian Journal of Ophthalmology 39, 183-5.

Cooper J \& Gianocotti F. (2014). Molecular insights into NF2/Merlin tumor suppressor function. FEBS Letters 588, 2743-2752.

Dossetor FM, Landau K \& Hoyt WF. (1989). Optic disk glioma in neurofibromatosis type 2 . American Journal of Ophthalmology $108,602-603$.

Duane TD, Schatz NJ \& Caputo AR. (1976). Pseudo-Duane's retraction syndrome. Transactions of the American Ophthalmological Society 74, 122-32.

Feucht M, Griffiths B, Niemüller I, Haase W, Richard G \& Mautner VF. (2008). Neurofibromatosis 2 leads to higher incidence of strabismological and neuro-ophthalmological disorders. Acta Ophthalmologica 86, 882-6.

Gittinger JW Jr, Hughes JP \& Suran EL. (1986). Medial orbital wall blow out fracture producing an acquired retraction syndrome. Journal of clinical neuro-ophthalmology. 6, 153-6.
Hee S, Hyung J, Yoon S \&Young S. (2009). A Case of Pseudo-Duane's Retraction Syndrome With Old Medial Orbital Wall Fracture. Korean Journal of Ophthalmology 23, 329-31.

Kaiser-Kupfer MI, Freidlin V \& Datiles MB. (1989). The association of posterior capsular lens opacities with bilateral acoustic neuromas in patients with neurofibromatosis type 2. Archives of Ophthalmology 107, 541-544.

Khan AO. (2005). Inverse globe retraction syndrome complicating recurrent pterygium. British Journal of Ophthalmology 89, 640-41.

Lew H, Lee JB, Kim HS \& Han SH. (2000). A case of congenital inverse Duane's retraction syndrome. Yonsei Medical Journal 41, 155-8.

Li X, Li J, Li J \& Zhen W. (2015). Schwannoma of the 6th nerve: case report and review of the literature. Chinese Neurosurgical Journal 1, 5.

Lida Y, Sakata K, Kobayashi N, Tatezuki J, Manaka H \& Kawasaki T. (2016). Orbital Abducens Nerve Schwannoma: A Case Report and Review of the Literature. NMC Case Report Journal 3, 107-109.

Murthy R. (2008). Inverse Duane's retraction syndrome following myocysticercosis. Indian Journal of Ophthalmology 56, 89-90.

Nakamura M, Carvalho GA \& Samii M. (2002). Abducens nerve schwannoma: a case report and review of the literature. Surgical Neurology 57, 183-8; discussion 188-9.

Pelit A. (2016). Pseudo-Duane Retraction Syndrome Secondary to Orbital Trauma: Case Report. International Journal of Ophthalmology and Clinical Research 3, 064.

Rato R, Correia M, Cunha JP \& Sutil P. (2012). Intraorbital Abducens Nerve Schwannoma. World Neurosurgery 78, 3/4:375. e1-375.e4.

Sivalingam A, Augsburger J, Perilongo G, Zimmerman R \& Barabas G. (1991). Combined hamartoma of the retina and retinal pigment epithelium in a patient with neurofibromatosis type 2. Journal of Pediatric Ophthalmology and Strabismus 28, 320-2.

Tonsgardf H \& Oesterle CS. (1993). The ophthalmologic presentation of NF2 in childhood. Journal of Pediatric Ophthalmology and Strabismus 30, 327-330.

Tung H, Chen T \& Weiss MH. (1991). Sixth nerve schwannomas. Report of two cases. Journal of Neurosurgery 75, 638-41.

Von Noorden GK. (1980). Binocular vision and ocular motility (Second Ed.). Mosby, St. Louis, USA. 